\title{
ASSOCIATION OF ESOPHAGEAL ACHALASIA AND PULMONARY ACTINOMYCOSIS INFECTION SIMULATING BRONCHIAL NEOPLASM
}

Iwao Takanami, Ken Takeuchi, and Masao Naruke, Tokyo, Japan

Pulmonary actinomycosis is a relatively uncommon disease caused by Actinomyces israelii, which is a normal inhabitant in the oral cavity and upper intestinal tract of human beings. It is believed that pulmonary actinomycosis enters the thorax via the bronchial tree by aspiration of contaminated secretions from the oropharynx. Achalasia of the esophagus is characterized by a high-grade obstruction at the esopha-

From the Department of Surgery, Teikyo School of Medicine, Tokyo, Japan

Received for publication Jan 26, 1999; accepted for publication March 12, 1999.

Address for reprints: Iwao Takanami, MD, Department of Surgery, Teikyo School of Medicine, 2-11 Kaga 2-Chome, Itabashi-Ku, Tokyo, 173 Japan.

J Thorac Cardiovasc Surg 1999;118:199-200

Copyright $(\odot 1999$ by Mosby, Inc.

$0022-5223 / 99 \$ 8.00+0 \quad \mathbf{1 2 / 5 4 / 9 8 5 8 6}$ gogastric junction. Pulmonary disease associated with achalasia is typically brought about by aspiration of contaminated matter from the dilated esophagus into the respiratory tree. Reported here is a case of achalasia complicated by pulmonary actinomycosis.

Clinical summary. A 52-year-old woman was admitted for cough and bloody sputum of 2 months' duration. She had a 3-year history of dysphagia, vomiting, and nocturnal aspiration of undigested food, which had yielded a diagnosis of achalasia of the esophagus. The chest radiograph showed consolidation in the apical and posterior segments of the right upper lobe. Thoracic computed tomographic scan revealed a mass of intrapulmonary soft tissue, without mediastinal involvement, and dilatation of the esophagus (Fig 1). Sputum smear and culture for acid-fast bacilli and sputum cytologic studies were negative on three occasions. Bronchoscopic examination revealed mucosal inflammation in the right upper lobe bronchi without any endobronchial lesion, and

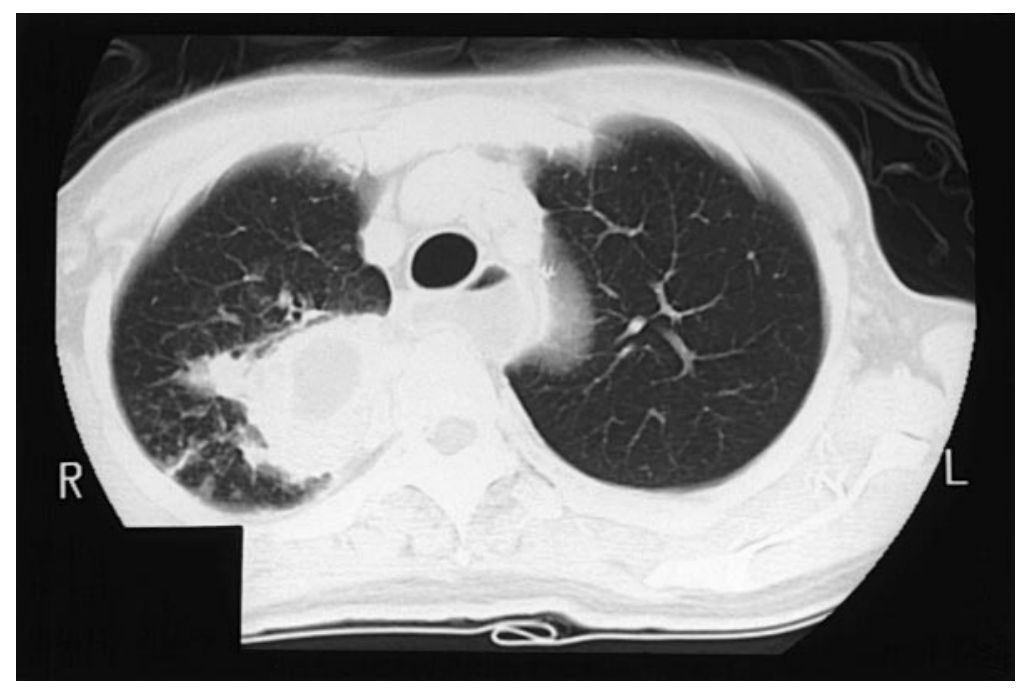

Fig 1. Computed tomograph of the thorax demonstrating an opacity and marked dilatation of the esophagus. 


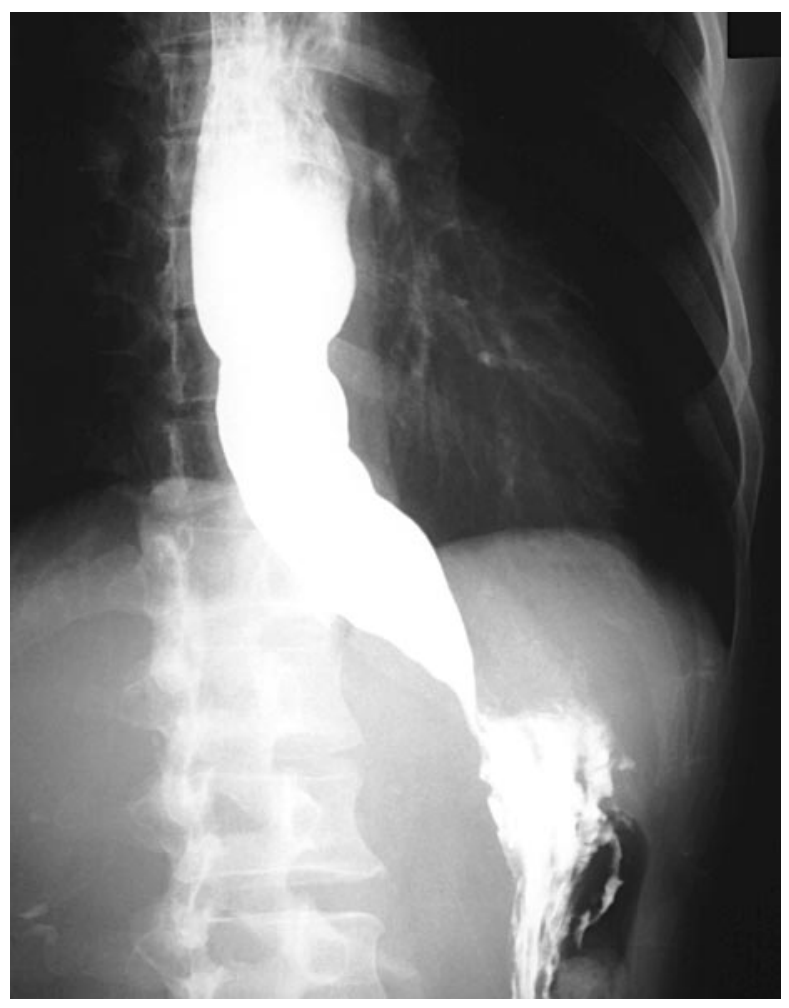

Fig 2. Film from barium examination showing a grossly dilated esophagus above a narrowed esophagogastric junction, limiting the amount of material that can pass into stomach.

examination of bronchial washing for acid-fast bacilli, fungus culture, and cytologic studies were all negative. A barium swallow showed marked dilatation of the esophagus with no peristalsis and failure of barium to flow into the stomach (Fig 2). The patient was initially treated orally with erythromycin. After 8 weeks the radiographic abnormality had not resolved, and bronchogenic carcinoma was considered as a likely cause of the lesion in the right upper lobe. Surgical exploration was then done. During the operation, a large mass was found, adherent to the pleura, and a right upper lobectomy was performed. Pathologic examination revealed necrotizing tissue in which Actinomyces organisms could be seen. The fresh specimen was sent for microbiologic examination, and Actinomyces israelii was isolated. After treatment with ampicillin, the patient made a good recovery, but she refused surgery for achalasia of the esophagus.

Discussion. The recurrent aspiration that often complicates achalasia causes chronic pulmonary inflammation impairing the lungs' ability to control infection effectively. Andersen,
Holman, and Olsen ${ }^{1}$ reported pulmonary symptoms in $10 \%$ of 601 cases of achalasia. The most extensive and most important type of aspiration pneumonia occurs when semicomatose or unconscious patients aspirate liquid gastric contents. However, even persons with apparently normal sensorium regurgitate and aspirate gastric contents during sleep, ${ }^{2}$ as seen in this patient with achalasia. Mycobacteria are frequently isolated from the sputum of bronchial secretions with achalasia and aspiration pneumonia. ${ }^{3}$ Reports have documented the association of fungal infection and aspiration pneumonia by esophageal disease, ${ }^{4}$ but little has been reported about the association of esophageal disease and pulmonary actinomycosis or aspiration pneumonia. Pulmonary actinomycosis is thought to be caused by aspiration of oral debris containing organisms normally inhabiting the oral cavity and upper intestinal tract of human beings. Actinomycosis of the upper intestinal tract has also been reported. ${ }^{5}$ Our patient with achalasia had a 3-year history of overflow aspiration and nocturnal (supine) aspiration. In this case, pulmonary actinomycosis is thought to have started after aspiration of contaminated oral or esophageal secretions by repeated vomiting resulting from achalasia. Pulmonary actinomycosis usually produces patchy alveolar infiltrates that may be unilateral or bilateral. Rarely, the infiltrates show pseudotumor, and there are reports of pulmonary actinomycosis simulating neoplasm of the lung. ${ }^{6}$ The diagnostic difficulty of pulmonary actinomycosis arises from a lack of specific findings. The clinical symptoms are common to many chest diseases, and the presence of Actinomyces organisms in the sputum is unhelpful, because it is a common oral communal. Chest radiographic appearances also vary. Although uncommon, pulmonary actinomycosis should be considered in the differential diagnosis of pulmonary neoplasm, particularly in atypical cases such as those with achalasia. Preoperative recognition would prevent the need for thoracotomy.

\section{REFERENCES}

1. Andersen HA, Holman CB, Olsen AM. Pulmonary complications of cardiospasm. JAMA 1953;151:608-12.

2. Berson W, Adriani J. "Silent" regurgitation and aspiration during anesthesia. Anesthesiology 1954;15:644-9.

3. Camera EJN, Lima JAC, Oliveira GB, Machado AS. Pulmonary finding in patients with chagasic megaesophagus: study of autopsied cases. Chest 1983;83:87-91.

4. Flora KD, Lieberman D, Schmidt W. Esophageal intramural pseudodiverticulosis. Dig Dis 1997;15:113-9.

5. Ng FH, Wong SY, Chang CM, Lai ST, Chan KY. Esophageal actinomycosis: a case report. Endoscopy 1997;29:133.

6. Shannon HM, Wightman AJA, Carey FA. Pulmonary actinomycosis-disguise. J Infect 1995;31:165-9. 